ISSN: 0213-2087 eISSN: 2444-7080

DOI: http://dx.doi.org/10.14201/shhc201836117130

\title{
NUEVAS DIMENSIONES DEL DISCURSO DE LOS DERECHOS HUMANOS
}

\section{New Dimensions of Human Rights Discourse}

\author{
Rafael de ASÍS \\ Universidad Carlos III de Madrid \\ rafael.asis@uc3m.es
}

Recepción: 27/04/2018 Revisión: 14/05/2018 Aceptación: 01/06/2018

RESUMEN: Aunque existen diferentes teorías de los derechos humanos, es posible señalar una serie de rasgos presentes en todas y cada una de ellas y que sirven para hablar de una teoría estándar de los derechos. En este trabajo describo dos tipos de retos diferentes que se plantean a esta teoría estándar de los derechos. Uno de ellos se mueve hacia el interior de la teoría y cuestiona algunos de sus rasgos. En este punto me referiré a las cuestiones del garante, de la tensión libertad-seguridad y de la universalidad. El otro gran reto cuestiona la validez de los derechos humanos como guía para el tratamiento de ciertos problemas. En este punto me referiré a los movimientos de personas y a las nuevas tecnologías. Ambos desafíos, que son una nueva muestra del carácter histórico que poseen los derechos humanos, obligan al discurso de los derechos a reaccionar e intentar resolverlos, aunque sea modificando alguno de los referentes que lo han caracterizado.

Palabras clave: derechos humanos; libertad; seguridad; universalidad; tecnologías.

ABSTRACT: Although there are different theories of human rights, it is possible to point out a series of features present in each and every one of them and that serve to speak of a standard theory of rights. In this paper I describe two different types of challenges that arise to this standard theory of rights. One of them moves into the interior of the theory and questions some of its features. At this point I will refer to the issues of the guarantor, of the freedom-security tension and of universality. The other great challenge questions the validity of human rights as a guide for the treatment of certain problems. At this point I will refer to the movements of people 
and new technologies. Both challenges, which are a sign of the historical character of human rights, force the discourse of rights to react and try to resolve them, even modifying some of the references that have characterized it.

Key words: human rights; freedom; security; universality; technologies.

Uno de los rasgos que individualizan a los derechos humanos es el de su dimensión internacional. En efecto, se trata de instrumentos cuya garantía y vigencia supera las fronteras nacionales. La dimensión internacional de los derechos tiene su punto de partida (dejando a un lado importantes precedentes) en 1948, con la aprobación de la Declaración universal de derechos humanos, y en 1966, con la aprobación de los Pactos Internacionales sobre Derechos Civiles y Políticos y sobre Derechos Económicos, Sociales y Culturales. Estos instrumentos, que configuran la Carta Internacional de Derechos Humanos, expresan un consenso ético, político y jurídico mundial ${ }^{1}$.

Y es que, si existe un acuerdo mundial sobre los referentes del discurso ético, jurídico y político, este está representado por los derechos humanos. Ahora bien, se trata de un acuerdo de mínimos que, además, es objeto de interpretaciones a veces contrapuestas.

Así, en el plano internacional, no es extraño encontrarse con decisiones de Organizaciones que nos parecen contrarias a los derechos, o con declaraciones de Estados radicalmente diferentes apoyándose ambas en los derechos humanos. Y algo parecido ocurre en el plano nacional donde, decisiones de los poderes públicos son cuestionadas por su posible violación de los derechos y donde todos los días se producen litigios entre particulares, que tienen su origen en diferentes maneras de entender los derechos.

Por otro lado, el catálogo y la fuerza de los derechos humanos ha sido siempre objeto de debate. Buena prueba de ello son las dificultades habidas a la hora de aprobar los Pactos Internacionales antes aludidos y que culminaron con la aprobación de dos instrumentos distintos ${ }^{2}$.

A pesar de ello, no cabe negar que los derechos funcionan como razones justificativas; su apelación obliga a realizar un esfuerzo extra a quien es acusado de violarlos y facilita el camino a quien puede demostrar que los satisface.

Así, a pesar de que existan diferentes interpretaciones sobre el significado de los derechos humanos, existen una serie de referentes que suelen estar presentes en cualquier discurso sobre los derechos humanos

1. Vid. Fernández Liesa, C.: El Derecho internacional de los derechos humanos en perspectiva bistórica. Madrid: Civitas, 2013, pp. 175 y ss.

2. Vid. al respecto SALVIOLI, F.: "Los derechos humanos civiles y políticos en el sistema universal de protección». En: AA. VV.: Historia de los derechos fundamentales, Tomo IV, Siglo XX, vol. III, libro I. Madrid: Dykinson, 2013, pp. 537 y ss.; y en la misma obra, SEATzu, F., "El Pacto Internacional de derechos económicos, sociales y culturales y la vigilancia de su aplicación», pp. 581 y ss. 
En la página del Alto Comisionado de Derechos Humanos se definen los derechos humanos en los siguientes términos:

Los derechos humanos son derechos inherentes a todos los seres humanos, sin distinción alguna de nacionalidad, lugar de residencia, sexo, origen nacional o étnico, color, religión, lengua, o cualquier otra condición. Todos tenemos los mismos derechos humanos, sin discriminación alguna. Estos derechos son interrelacionados, interdependientes e indivisibles. Los derechos humanos universales están a menudo contemplados en la ley y garantizados por ella, a través de los tratados, el derecho internacional consuetudinario, los principios generales y otras fuentes del derecho internacional. El derecho internacional de los derechos humanos establece las obligaciones que tienen los gobiernos de tomar medidas en determinadas situaciones, o de abstenerse de actuar de determinada forma en otras, a fin de promover y proteger los derechos humanos y las libertades fundamentales de los individuos o grupos ${ }^{3}$.

Esta definición recoge una idea estándar de los derechos que puede completarse añadiendo cuál es su función. Si buscamos en el ámbito de Naciones Unidas, encontramos que se afirma que los derechos humanos «son garantías esenciales para que podamos vivir como seres humanos», y continúa afirmando que sin ellos «no podemos cultivar ni ejercer plenamente nuestras cualidades, nuestra inteligencia, talento y espiritualidad ${ }^{4}$. Y de este modo, la Declaración Universal de Derechos Humanos, comienza su Preámbulo afirmando: "Considerando que la libertad, la justicia y la paz en el mundo tienen por base el reconocimiento de la dignidad intrínseca y de los derechos iguales e inalienables de todos los miembros de la familia humana».

Pues bien, a pesar de que, como he señalado al comienzo, existen diferentes formas de entender los derechos humanos, todas ellas comparten una serie de referentes separándose en el peso que dan a estos y, en su caso, en aquellos otros rasgos que les acompañan ${ }^{5}$. De esta forma, por ejemplo, difícilmente encontraremos una teoría de los derechos humanos que no los conciba como instrumentos derivados de la dignidad humana y destinados a favorecer el desarrollo de una vida en libertad e igualdad.

Para entender los derechos humanos hay que presumir la valía de lo humano, de la persona como agente moral dotado de una dignidad derivada de su excelencia y con una vida orientada a la consecución de un plan de vida. Los derechos son inherentes a todas las personas, universales, interrelacionados, interdependientes e indivisibles. En este sentido se tienen con independencia de cualquier condición y circunstancia, todos ellos están conectados y la insatisfacción de uno afecta a la de otro, por lo que no pueden garantizarse unos y no otros. Los derechos humanos

3. En <http://www.ohchr.org/SP/Issues/Pages/WhatareHumanRights.aspx>, consultada el día 24 de febrero de 2017.

4. En <http://www.un.org/es/rights/overview/>, consultada el 24 de febrero de 2017.

5. Vid. PeCEs-BARba, G. et al.: Curso de derechos fundamentales. Madrid: BOE-Universidad Carlos III, 1995. 
constituyen verdaderas obligaciones para los Estados y necesitan del Derecho, del Estado de Derecho y de la Democracia, para su realización completa. La protección de los derechos permite una convivencia social en paz y en libertad, favorecedora del desarrollo de las personas.

Esta concepción de los derechos se ha construido desde el Tránsito a la Modernidad $^{6}$ y tiene su origen en tres grandes reflexiones que aún hoy perviven: la reflexión sobre los límites al Poder político, la reflexión sobre la tolerancia y la reflexión sobre la humanización del Derecho penal y procesal. Estas tres reflexiones son las que están detrás de los primeros textos sobre derechos humanos.

A partir de ese momento, se habla de cuatro grandes procesos que han caracterizado la historia de los derechos: el de positivación, el de generalización, el de internacionalización y el de especificación.

El proceso de positivación supone el paso de los derechos desde el plano de la reflexión al plano del Derecho, y se expresa en tres contextos geográficos concretos: el inglés, el francés y el norteamericano. En este sentido, implica la incorporación de los derechos al Derecho junto con el establecimiento de un sistema de garantías. Por su parte, el proceso de generalización surge como respuesta al primer reconocimiento de los derechos que está asociado a una clase social concreta. En este sentido, implica la extensión de la titularidad y de la satisfacción de los derechos al conjunto de la ciudadanía. El proceso de internacionalización tiene su origen en la necesidad de controlar el poder de los Estados y en la existencia de cuestiones que afectan a los derechos que superan las fronteras de un Estado. Implica el reconocimiento de los derechos y el establecimiento de un sistema de garantías en el ámbito internacional. Por último, el proceso de especificación tiene su origen en la aparición de propuestas y de textos jurídicos que se caracterizan por predicar la existencia de derechos específicos de un grupo.

De esta forma, en la actualidad, los derechos humanos se conciben como herramientas éticas, políticas y jurídicas. Como herramientas éticas, protegen la dignidad de los seres humanos; como herramientas políticas sirven como criterios de legitimidad a los Estados y a las Instituciones; como herramientas jurídicas llevan a cabo dos grandes funciones, la objetiva y la subjetiva. A través de la primera los derechos funcionan como criterios de validez del Ordenamiento jurídico; a través de la segunda funcionan como herramientas para proteger la libertad.

De alguna, estas dimensiones de lo que podemos denominar como teoría o discurso estándar de los derechos, surgen en el siglo xx y son las que están detrás de la Carta Internacional de los Derechos Humanos. La teoría estándar de los derechos es el resultado de la evolución histórica de estos instrumentos y de su proyección (y adaptación) en los distintos problemas que han ido acompañando a los seres humanos.

6. Vid. Peces-Barba, G., Fernández, E., De Asís, R. y Ansuátegui, F. J. (dirs.): Historia de los derechos fundamengtales, T. I, Tránsito a la Modernidad. Madrid: Dykinson, 2003. 
De esta manera, la teoría de los derechos ha ido respondiendo a diferentes problemas y retos, y al hilo de estas respuestas ha ido evolucionando y transformándose.Pues bien, en este trabajo me voy a referir a dos tipos de retos diferentes que se plantean a la que vengo denominando como teoría estándar de los derechos humanos. Uno de ellos se mueve hacia el interior de la teoría y cuestiona algunos de sus rasgos; el otro se mueve hacia el exterior y cuestiona su propia validez como guía para el tratamiento de ciertos problemas. Ambos desafíos, que son una nueva muestra del carácter histórico que poseen los derechos humanos ${ }^{7}$ obligan al discurso de los derechos a reaccionar e intentar resolverlos, aunque sea modificando alguno de los referentes que lo han caracterizado.

\section{Retos de la Teoría de los derechos hacia El interior}

Lo que denomino retos hacia el interior son problemas que se proyectan en elementos constitutivos de la teoría de los derechos. En este trabajo me referiré brevemente a tres. El primero, el del garante, es predominantemente jurídico-político; el segundo, el de la tensión libertad-seguridad, es predominantemente ético político; el tercero, el de la universalidad, es predominantemente ético-jurídico.

Cuando hablo de garante estoy haciendo referencia a la institución o poder encargado de hacer valer los derechos ${ }^{8}$. En este sentido, tradicionalmente, la cuestión del garante de los derechos se desdobla en dos momentos. El primero es el momento del desarrollo, de la legislación; el segundo es el momento del control de su desarrollo y de su aplicación. Esta lógica presente en la teoría de los derechos supone reconocer cierta indeterminación en su significado, que el legislador se encarga de concretar, pero no una indeterminación absoluta, que dejaría sin sentido el momento del control. Además, la manera de resolver esta lógica que se traduce normalmente en vincular el desarrollo con el Parlamento y el control con el Juez (nacional, constitucional o internacional), expresa también una opción sobre la legitimidad de estos garantes, que se basa, en un primer momento en el principio democrático y en un segundo momento en el principio de la imparcialidad. No se trata de la única opción sobre la legitimidad del garante presente en la teoría de los derechos. Así, si observamos los requisitos que se exigen para formar parte de estas instancias, veremos que hay algunos criterios que funcionan siempre, entre los que destaco el del conocimiento jurídico y el de la nacionalidad.

Como se habrá podido ya imaginar, aun aceptando la visión que la Teoría de los derechos humanos estándar maneja, es posible que alguno de los requisitos anteriores pueda hacer poco coherente a la teoría. En este sentido, es posible

7. Vid. Peces-Barba, G.: "Sobre el puesto de la historia en el concepto de los derechos fundamentales", eAnuario de derechos bumanos, n. 4, 1986-1987, pp. 219 y ss.

8. Vid. De Asís, R.: "La garantía del Estado Constitucional». En: GarRido, I. y EsPinOza DE LOs Monteros, J.: Paradigmas y desafíos del constitucionalismo contemporáneo. Granada: Comares, 2014 , pp. 71 y ss. 
justificar como opción razonable el que, como existe un margen de discrecionalidad en el desarrollo del derecho, este quede en manos de un órgano compuesto por, entre otros requisitos, nacionales del país en cuestión. Pero resulta más difícil justificar mantener esta exigencia cuando, según esa visión, ya no hay discrecionalidad, esto es, en el momento del control. Y mucho menos cuando ese control se hace en el ámbito internacional.

El problema se hace más evidente cuando no se comparte esa visión de la interpretación y se considera, por ejemplo, que en la interpretación de los derechos siempre hay márgenes de discrecionalidad, que se trata de una actividad ética y política. En este caso, puede llegarse a cuestionar la necesidad de ser jurista para estar en instancias de control o también puede llegarse a proponer la importancia de incorporar, en sociedades plurales, cuotas que representen la diversidad en esas mismas instancias de control.

Me parece necesario que la Teoría de los derechos humanos reflexione sobre los criterios de legitimidad de sus garantes, proponiendo aquellos que sean aceptables y coherentes.

El segundo de los retos es el de la tensión entre libertad y seguridad 9 . Como ya he señalado, una de las reflexiones que están en el origen de la idea de los derechos humanos es aquella que se proyecta en la necesidad de humanizar el Derecho penal y procesal. El Derecho penal se ha presentado en el mundo moderno como uno de los principales instrumentos para la protección de los derechos, a través de la consideración como delitos de aquellas acciones y omisiones que los vulneran. En todo caso, la utilización del Derecho penal en el ámbito de los derechos trata de compaginar la necesidad de responder frente a las transgresiones de los derechos con el respeto a los valores y principios que presiden su discurso. De ahí que hasta época reciente la utilización del Derecho penal se haya desenvuelto dentro de un marco teórico entre cuyos referentes están: a) la consideración de las normas penales como el último recurso para la garantía de los derechos (lo que se ha denominado como el Derecho penal mínimo); b) la exigencia del respeto a los derechos de aquellos que delinquen mediante la satisfacción del derecho a la tutela judicial efectiva y mediante la prohibición de todo tratamiento que vulnere sus derechos; c) la consideración de que el fin de la pena deber ser la reinserción social del delincuente.

No obstante, en la actualidad, puede percibirse un cuestionamiento de este marco que puede llegar a implicar un paso atrás en la evolución de los derechos. Este cuestionamiento tiene dos grandes hitos. Por un lado, la aparición de nuevas concepciones sobre el Derecho penal, entre las que destaca la denominada como el Derecho penal del enemigo, que supone defender la posibilidad de utilizar dos varas de medir los comportamientos de los individuos; por un lado, la de los considerados como enemigos (por enfrentarse a los principios y valores del sistema

9. Vid. al respecto, Revenga, M. (dir.): Terrorismo y derecho bajo la estela del 11 de septiembre. Valencia: Tirant Lo Blanch, 2014, pp. 13 y ss. 
constitucional a través de comportamientos que se consideran como especialmente dañinos) y la de los ciudadanos en general. Por otro, la justificación de prácticas que había pasado ya a formar parte del museo de la historia, como la tortura ${ }^{10}$. Una de las causas que han llevado a este cuestionamiento es la aparición del terrorismo internacional cuyo momento álgido fueron los atentados de las torres gemelas del 11 de septiembre.

De esta forma, otro de los desafíos actuales a la teoría de los derechos consiste en encontrar una respuesta adecuada a la tensión entre libertad y seguridad; respuesta que permita enfrentarse a nuevos fenómenos sin traicionar la idea de los derechos. Un lugar importante en el tratamiento de este reto está ocupado por la necesaria atención a la situación y a los derechos de las víctimas ${ }^{11}$.

El tercero de los retos se relaciona con una de las críticas más conocidas a la teoría de los derechos humanos y que se centra en subrayar que se trata de una teoría ética propia de occidente. En otros trabajos me he referido ya a este cuestionamiento ${ }^{12}$.

Puede parecer raro que sitúe este problema en los retos hacia el interior. Me interesa aquí destacar como, una de las proyecciones de esta crítica coincide con la puesta en entredicho del rasgo de la universalidad.

Como es sabido, el rasgo de la universalidad de los derechos se defiende tanto desde un punto de vista descriptivo, los derechos humanos son universales, cuanto desde un punto de vista prescriptivo, los derechos humanos deben ser universales. En ambos planos, la universalidad supone el que todas las personas son titulares de derechos con independencia de cualquier circunstancia. Y esto normalmente se defiende, como hemos visto en la teoría estándar, desde un modelo de ser humano que posee un carácter universal, esto es, desde una visión universal de lo humano, que suele ser descrita en términos de capacidad y excelencia, frente al resto de seres vivos. Además, en el caso de los derechos, la universalidad se proyecta también en la defensa de un modelo de sociedad, con valor universal, que favorece el desarrollo de ese modelo humano.

Es en este marco teórico en el que aparece la dignidad como expresión del valor que posee lo humano y como exigencia de la obligación que tenemos de respetarlo. Y es también en este marco teórico en donde la idea de igualdad, como exigencia de trato idéntico y de igual reconocimiento, se convierte en eje central del discurso.

Pues bien, esta idea de universalidad es puesta en entredicho tanto en el plano descriptivo, cuanto en el prescriptivo.

10. Vid. LA Torre, M.: "Amistades peligrosas. Tortura y Derecho", Derechos y Libertades, n. 28, 2013, pp. 25 y ss.

11. Vid. Rodríguez Uribes, J. M.: «Sobre el terrorismo y sus víctimas", Derechos y Libertades, n. 27, 2012, pp. 241 y ss.

12. Vid. De Asís, R.: Cuestiones de derechos. Bogotá: Universidad Externado de Colombia, 2005, pp. 23 y ss. 
En el primero de ellos se afirma que los derechos no son universales ni lo han sido nunca. Para ello se utilizan ejemplos que tienen que ver con la distinción entre nacionales y extranjeros, entre mayores y menores, o entre personas con capacidad jurídica y personas sin capacidad jurídica.

Esta crítica trata de pasar por la realidad a la teoría de los derechos y se proyecta sobre alguno de los otros rasgos que veíamos en la teoría estándar, como por ejemplo el de la indivisibilidad. En esta línea puede afirmarse que los derechos nunca han sido indivisibles en el ámbito jurídico sino todo lo contrario; el diferente tratamiento generalizado entre derechos civiles y derechos económicos, sociales y culturales es buena prueba de ello.

Pero la idea de universalidad también se critica en su dimensión prescriptiva. Así, se cuestiona tanto en lo que tiene que ver con el reconocimiento de derechos cuanto en lo que atañe con el resto de dimensiones que hemos apuntado. Se afirma que es posible defender que los derechos no deben ser universales, que no existe una idea de lo humano universal ni un modelo de sociedad universalmente valioso.

Dos de las dimensiones de este cuestionamiento se proyectan sobre dos de los valores que se constituyen en referentes de la teoría estándar de los derechos: la dignidad y la igualdad.

Como es sabido, la justificación de la dignidad humana ha seguido en la historia dos caminos ${ }^{13}$. Uno de ellos, de corte religioso, ha basado la dignidad humana en la idea del ser humano hecho a imagen y semejanza de la autoridad divina; el otro, de índole racional, ha justificado la dignidad humana en la consideración del ser humano como ser racional, capaz de razonar, de realizar juicios estéticos, etc. Este segundo camino es el que está en el origen del discurso moderno de los derechos humanos y se incorpora a su teoría estándar. Desde él se ha subrayado un modelo de ser humano, como ser superior, basado en las capacidades, que ha estigmatizado todo aquello que se sale de ese modelo. Un ejemplo está constituido por las personas con discapacidad.

Los actores del discurso de los derechos son aquellos que superan un estándar no siempre explícito de supuesta racionalidad: las personas con capacidad ${ }^{14}$. Los que no poseen la racionalidad exigida, aquellos que presentan alguna discapacidad de raciocinio (temporal o permanente), no son considerados como sujetos del discurso (y sí, en todo caso, objetos) ${ }^{15}$, quedando así su horizonte moral pendiente de la decisión de los capaces ${ }^{16}$. Y esto ha provocado que el tratamiento de estas personas sea una cuestión de solidaridad y no necesariamente de derechos y,

13. Vid. Pele, A.: "Una aproximación al concepto de dignidad humana", Universitas. Revista de Filosofía, Derecho y Política, n. 1, 2004-2005, pp. 9 y ss.

14. Vid. De Asis, R.: Sobre discapacidad y derechos. Madrid: Dykinson, 2013, pp. 36 y ss.

15. Rawls, J.: El liberalismo político, trad. de A. Domenech. Barcelona: Crítica, 1996, p. 105.

16. Carlson, L.: "Pihilosophers of intellectual disability: a taxonomy». En: KitTAy, E. y CarLSON, L. (eds.): Cognitive Disability and its Challenge to Moral Philosophy. Oxford: Willey-Blackwell, 2010, pp. 315 y ss. 
además, que sobre ellos no funcione la relación respeto propio-reconocimiento social. Solo recientemente es posible hablar de un cambio que se percibe claramente en el campo del tratamiento del menor y que comienza a percibirse, de forma mucho más tímida, en el tratamiento de las personas con discapacidad, consistente en adoptar un enfoque de derechos.

En lo referente a la igualdad, hay que advertir que el tratamiento estándar de la igualdad ha implicado asumir que el trato igual está en línea de principio justificado y no así el trato diferente. En efecto, la idea de igualdad más extendida y aceptada ha sido aquella que la identifica con el trato igual generalizado, esto es con la igualdad formal. Así, para que se acepte un trato diferente deben aportarse razones y esto no ocurre con el trato igual. Hemos asumido e integrado en el discurso de los derechos la idea de que los seres humanos somos iguales y debemos ser tratados de manera igual.

Pues bien, es posible afirmar que, en la actualidad, el discurso de los derechos en materia de igualdad debe partir del hecho de la diferencia, de manera contraria a lo que ocurrió en el origen de la historia de los derechos (y todavía hoy se mantiene por algunos) en donde se hablaba de la igualdad como un hecho que caracterizaba a los seres humanos. Los seres humanos somos diferentes y nos encontramos en situaciones diferentes. Y a partir de ahí, el discurso sobre la igualdad se desenvuelve a través de dos grandes proyecciones: la diferenciación negativa y la positiva. En la primera, la diferenciación negativa, se trata de averiguar qué rasgos de los que nos hacen diferentes o qué situaciones que nos diferencian, son irrelevantes para justificar un trato distinto; en la segunda, la diferenciación positiva, se trata de averiguar qué rasgos de los que nos hacen diferentes o qué situaciones que nos diferencian, son relevantes para justificar un trato distinto.

\section{Retos de LA TEORÍA DE LOS DERECHOS HACIA EL EXTERIOR}

Los retos de la teoría de los derechos hacia el exterior son problemas que acontecen a los derechos y que, de nuevo, dan cuenta de su dimensión histórica. En este punto, voy a destacar dos muy diferentes y que en la actualidad poseen una singular importancia. Por un lado, me referiré a los movimientos de personas y, por otro, a las nuevas tecnologías.

Al igual que ocurría en el tratamiento de los derechos de otras personas o colectivos en situación de vulnerabilidad, hace unos años, la reflexión sobre los derechos de los inmigrantes se había llegado a proyectar sobre la justificación o no de reconocer derechos específicos. Ciertamente, y es importante aclararlo, esto no significaba que en la realidad la cuestión de la equiparación de derechos estuviera resuelta, ni que la aproximación a la inmigración no se hiciera de manera sesgada.

Desde siempre la reflexión sobre los derechos de los inmigrantes se condicionó (se condiciona) por la manera en la que se dotaba de contenido al propio término de inmigrante, y por la manera en la que se interpretaba (se interpreta) el derecho a la libre circulación en el ámbito internacional. En efecto, como muchos 
han insistido, el término inmigrante se ha utilizado siempre en el lenguaje jurídico y político, para hacer referencia a un tipo concreto de persona que viene de fuera pero no a toda persona que viene de fuera. En el ámbito de la extranjería ${ }^{17}$ el término se utiliza para referirse a extranjeros que se desplazan en condiciones económicas especialmente bajas o como consecuencia de persecuciones y no para aquellos que lo hacen por razones laborales, por turismo, etc. Por otro lado, la cuestión de la inmigración, también desde siempre, ha sido analizada partiendo de un punto de vista sobre los derechos que, igualmente, ha condicionado todo el discurso y que tiene su origen en una cuestionable manera de interpretar el artículo 13 de la Declaración Universal de Derechos Humanos. Este artículo establece: «1. Toda persona tiene derecho a circular libremente y a elegir su residencia en el territorio de un Estado. 2. Toda persona tiene derecho a salir de cualquier país, incluso el propio, y a regresar a su país». Pues bien, este precepto reconoce la libertad de movimiento y residencia en el interior de un Estado, así como el derecho de toda persona a abandonar cualquier país (incluido el suyo) y a retornar al país del que se es ciudadano. Sin embargo, se considera que no reconoce el derecho concomitante a entrar o residir en cualquier país del que no se es ciudadano. Como ha señalado A. M. López Sala: "El derecho de entrada se encuentra supeditado al principio de autodeterminación colectiva de una comunidad nacional y así se reconoce -es un principio no disputado- en el Derecho internacional» ${ }^{18}$.

En todo caso, y a pesar de lo anterior, como decía, existía un cierto consenso, al menos en el interior del discurso de los derechos, en lo referido a qué política de gestión de las migraciones era la que más favorecía los derechos ${ }^{19}$, en defender el mayor grado de generalización e, incluso, en la necesidad de un cierto reconocimiento de derechos específicos. Lo que se tenía claro es que un modelo de gestión de las migraciones basado en la exclusión era contrario a los derechos y también, que la evolución de los derechos, al hilo del llamado proceso de generalización, debía ir dirigida a lograr el mayor grado posible de equiparación entre los derechos de los nacionales y de los extranjeros.

En la actualidad, hemos dado un paso atrás muy importante que implica en muchos casos, una ausencia de satisfacción de los derechos más básicos. La crisis migratoria presente en Europa, y la vergonzosa política europea y de muchos de los países implicados dan buena cuenta de ello. Como también lo hace, en España, la situación de los inmigrantes en los Centros de Internamiento de Extranjeros ${ }^{20}$.

17. Vid. BADE, K. J. (comp.): Población, trabajo y migración en los siglos XIX y XX en Alemania, trad. de J. A. Colás Sánchez. Ministerio de Trabajo y Seguridad Social, 1992.

18. López SAla, A. M.: "Los retos políticos de la inmigración", Isegoría, 26, 2002, p. 92.

19. JiménEz, C.: «Modelos sociopolíticos e ideológicos ante la diversidad cultural: la propuesta intercultural». En: Alcina, J. y CALÉs, M. (eds.): Hacia una ideología para el siglo XXI. Ante la crisis civilizatoria de nuestro tiempo. Madrid: Akal, 2000, pp. 132 y ss.

20. De LuCAs, J.: "La herida original de las políticas de inmigración. A propósito del lugar de los derechos humanos en las políticas de inmigración", Isegoría, 26, 2002, p. 72. 
Sorprendentemente no existe un enfoque de derechos ante esta crisis. Incluso en materia de derechos de los niños y niñas afectados por esta crisis, el enfoque de derechos, que exigiría medidas basada en el interés superior del menor, y no en la seguridad o en el orden público, está ausente. Y subrayo lo de sorprendentemente porque este enfoque debería ser el apropiado teniendo en cuenta que estamos hablando de Europa, esto es, del territorio en el que supuestamente se inicia la historia de los derechos humanos (aunque también es cierto que, las dificultades existentes para dotar de fuerza institucional a los derechos humanos en el proceso de construcción de la Unión Europea, ya ponían de manifiesto esta especie de paradoja).Tal vez, la razón de que no se utilice este enfoque tenga que ver con las debilidades e incoherencias de la teoría estándar de los derechos. Así, por ejemplo, existen tres grandes rasgos de la teoría de los derechos humanos que resultan cuando menos desmentidos cuando se proyectan en la inmigración: la idea de que los derechos humanos son límites al poder, la idea de que los derechos humanos están fuera del regateo político y el rasgo de la universalidad. Sobre este último ya he tenido ocasión de detenerme.

La construcción de los derechos como límites al Poder ha llegado a significar que los derechos están por encima de las mayorías, esto es, que los derechos son los límites de las decisiones políticas presuntamente legítimas (las democráticas). Sin embargo, vemos día tras día como los derechos están en manos del poder político (es posible que esto sea irremediable), pero de un poder político real, concreto, en forma de Estado. Y es que una de las bases de la teoría de los derechos humanos lo constituye la idea de soberanía y con ella la idea de nacionalidad. Idea que sirve para construir la diferenciación entre nacional y extranjero, y para justificar, de manera muy cuestionable, la limitación de los derechos humanos a estos últimos.

La segunda de las ideas, la de la ausencia del regateo político es también desmentida por la realidad. Ciertamente lo ha sido siempre. Buena cuenta de ello es la presencia del principio de reciprocidad en el ámbito de los derechos de los extranjeros (cuya tradición se remonta al Código de Napoleón). Pero lo es hoy más de forma descarada con el copago en materia de asilo y refugio (instaurado en determinados países) o las políticas de expulsión o trueque, también en esta materia, y la consiguiente mercantilización de las personas.

El discurso de los derechos humanos debe hacerse coherente y afianzarse en los contextos nacionales e internacionales, respondiendo firmemente a situaciones nacionales e internacionales que claramente lo transgreden. Las limitaciones a los derechos deben estar basadas en argumentos éticos coherentes con los valores y principios que presiden este discurso, y la nacionalidad o la economía, por sí mismas, no son argumentos de este tipo. Es necesario construir un sistema político global que esté al servicio de los derechos y en el que la solidaridad y la igualdad desempeñen un papel fundamental.

Esta construcción política global basada en los derechos y la solidaridad debe modificar el rumbo seguido por otra de las patas de las políticas migratorias como es la de las relaciones entre los Estados. 
Ciertamente el logro de lo anterior requiere, como vengo subrayando, de un cambio en la teoría de los derechos y en la determinación de su alcance. Y algo parecido sucede cuando relacionamos el discurso de los derechos con los avances tecnológicos y con las tecnologías emergentes.

El término de tecnologías emergentes se utiliza para referirse a la combinación de tecnologías transformativas como la nanociencia y la nanotecnologia; la biomedicina y la ingeniería genética; la tecnología de la información; las ciencias $\operatorname{cognitivas}^{21}$. El uso conjunto de estas tecnologías recrudece algunos de los problemas «clásicos» que plantean los avances científicos y tecnológicos y pone sobre la mesa nuevos retos ${ }^{22}$.

En este sentido, la necesidad de realizar una regulación jurídica de las tecnologías emergentes es evidente, si bien no existe una posición unánime sobre cómo debe ser ni cuales deben ser los referentes desde los que construir esta regulación.

Para algunos, los "bioconservadores", las tecnologías emergentes son un verdadero peligro; para otros, los "transhumanistas", son una verdadera oportunidad; los «neurodeterministas» utilizan estos avances para demostrar que la ética, tal y como la hemos construido, no existe...

La posición de los bioconservadores se basa en el daño que los avances tecnológicos y, sobre todo, algunas propuestas sobre su aplicación pueden producir a la humanidad y al medio ambiente ${ }^{23}$. Para ellos, el bien no puede definirse en términos de manipulación de la vida, ya que esta siempre es algo negativo. Se oponen al uso de la tecnología para modificar la naturaleza humana ya que consideran que esto minará nuestra dignidad ${ }^{24}$. Pretenden preservar los atributos individuales que definen al ser humano, conservar al individuo como especie y mantener lo que sería su evolución natural. Y por eso, se hace necesario renunciar a ciertas tecnologías y en todo caso manejar el principio de precaución. Los seres humanos de hoy no tienen derecho de modificar el futuro ni de predeterminar cómo serán las personas del mañana. No tenemos derecho a jugar a ser Dios.

Por su parte, el neurodeterminismo es un planteamiento que reduce la moral a la biología. Así, se defiende que nuestra estructura ética tiene un componente biológico que nos hace singulares frente al resto de los seres vivos ${ }^{25}$. En todo caso, dentro del neurodeterminismo nos encontramos con distintas posiciones. Algunos rechazan el libre albedrío y la autonomía considerando al yo como un estado del

21. Se utiliza el término NBIC (nano, bio, info y cogno) para hacer referencia a la combinación de nanotecnología, la biomedicina, la informática y la neurociencia.

22. Me he referido a esta cuestión en Una mirada a la robótica desde los derechos humanos. Madrid: Dykinson, 2015, pp. 23 y ss.

23. Sobre los peligros de los avances tecnológicos y la necesidad de utilizar bien el progreso vid. Rees, M.: Nuestra hora final: ¿será el siglo XXI el último de la humanidad? Barcelona: Crítica, 2004.

24. Vid. Fukuyama, F.: Our Posthuman Future: Consequences of the Biotechnology Revolution. New York: Strauss and Giroux, 2002, p. 149.

25. Vid. Damasio, A. R.: Y el cerebro creó al hombre. ¿Cómo pudo el cerebro generar emociones, sentimientos ideas y el yo? trad. de Ferrán Meler Orti. Barcelona: Destino, 2010. 
cerebro. En este sentido han llegado a proponer una nueva ética, una nueva filosofía de la vida basada en los datos cerebrales, una moral que tiene su origen en la biología del cerebro ${ }^{26}$. No obstante, otras direcciones defienden que no estamos completamente determinados por los procesos físicos. El libre albedrio y el sentido de la responsabilidad no surgen solo de un hemisferio cerebral sino también de la interacción social, de la vida en comunidad.

Para el transhumanismo el fin de la humanidad es inevitable si no se aprovecha el desarrollo de la ciencia y la tecnología, consiguiendo instrumentos y aparatos que permitan luchar contra ese fin. Y ese desarrollo sin límites permitirá crear máquinas singulares que puedan fusionarse con los seres humanos. Así, el transhumanismo defiende así la libertad del ser humano de utilizar la tecnología para mejorar su vida y la de los demás, para ser más fuertes, más longevos, menos violentos... Los transhumanistas creen que las tecnologías de mejora humana deben ser ampliamente difundidas, que las personas deben elegir cuál de estas tecnologías se aplican a sí mismos, y que los padres deben tener el derecho de elegir las mejoras para sus hijos.

Se trata de un movimiento que posee distintas corrientes ${ }^{27}$ y que ha sido considerado por algunos como peligroso por atentar contra la esencia de lo humano. Así, algunas posiciones transhumanistas se enfrentan a las éticas humanistas, si bien tal vez sea mejor considerarlas como parte del posthumanismo. Pero muchos de los defensores de esta corriente aplican relecturas de principios o valores modernos y se presentan como un paso lógico del humanismo antes los cambios científicos y tecnológicos.

En definitiva, el transhumanismo rechaza la visión de la naturaleza como algo constante e inalterable y defiende que el valor moral del ser humano no se produce por pertenecer a una especie sino por lo que hace. Por eso, los avances tecnológicos deben ser utilizados para la mejora moral de los humanos ${ }^{28}$. Se trata de una mejora que no producirá efectos negativos ya que si supone mejorar el comportamiento moral de las personas difícilmente se podrá pensar en que se hace un mal moral ${ }^{29}$.

De estos tres planteamientos, el bioconservadurismo suele ser el posicionamiento más cercano a los derechos humanos, al defender una ética pública que asume entre sus primeros principios el imperativo categórico que H. Jonas ha

26. Vid. Churchland, P. S.: El cerebro moral. Barcelona: Paidós, 2012. Para Churchland, la conciencia es consecuencia de distintos fenómenos, muchos de ellos neurobiológicos.

27. Así se habla de "anarco-transhumanismo", "transhumanismo cristiano", "transhumanismo democrático», "socialismo transhumanista». Una historia del transhumanismo puede consultarse en BosTrom, N.: "Una historia del pensamiento transhumanista", Argumentos de razón técnica: Revista española de ciencia, tecnología y sociedad, y filosofía de la tecnología, n. 14, 2011, pp. 157 y ss.

28. Vid. Persson, I., y Savulescu, J.: "Moral Transhumanism», The Journal of Medicine E Philosophy, vol. 35, 2010, pp. 656 y ss.

29. Vid. Persson, I.: "Could it be permissible to prevent the existence of morally enhanced people?», The Journal of Medicine E Philosophy, vol. 38, 2012, pp. 692 y 693. 
considerado como propio de la sociedad del siglo xxI: "Obra de tal modo que los efectos de tu acción sean compatibles con la permanencia de una vida humana auténtica en la Tierra ${ }^{30}$.

Ahora bien, la teoría de los derechos debe desenvolverse en un marco ético abierto al progreso tecnológico y científico lo que supone no despreciar completamente las tesis neurodeterministas ni transhumanistas. No debemos renunciar a los avances de la tecnología y a su utilización para la mejora de la vida humana, aunque esto nos obligue a matizar el antropocentrismo y sustituirlo por una visión ecocéntrica y biocéntrica, que implica el respeto a la vida y al ser en su conjunto ${ }^{31}$.

Este marco ético debe, por otro lado, atender a nuestra dimensión social, ser consciente de la importancia de las relaciones interpersonales para la consecución de una vida humana digna, configurando así una ética que tenga en cuenta al otro. El ser humano está ligado a la actividad de los demás por lo que los otros ocupan un lugar indispensable. Formamos parte de una cultura y de una sociedad que nos transforma. El propio cerebro está equipado para absorber y aplicar los aprendizajes que se extraen de lo que nos rodea (principalmente otros seres humanos). De esta forma, según K. Evers, es posible manejar una concepción del cerebro que no lo considere como «un autómata biológico rígido ni como un ordenador desprovisto de emociones, sino como un órgano emocional, dinámico y variable, activo de manera autómata (de modo consciente y no consciente) y que ha evolucionado en una simbiosis sociocultural-biológica "32.

Es igualmente necesario que este marco ético preste atención a las generaciones futuras poniendo especial énfasis en la responsabilidad y la previsión, en una responsabilidad orientada hacia el futuro que nos permite progresar con cautela 33 .

Por último, este marco ético debe abordar la cuestión de la discapacidad. Se trata de una cuestión que se presenta como uno de los grandes asuntos a la hora de enfrentarnos a los retos de las tecnologías emergentes. Por eso, es importante estar atentos a la evolución de esta reflexión y al papel que en ella desempeña la discapacidad $^{34}$.

30. Jonas, H.: El principio de responsabilidad. Barcelona: Herder, 1995, p. 40.

31. Vid. Rivero Weber, P.: "Ciencia y Técnica: Hacia un nuevo humanismo bioético». En: Álvarez Del Río, A. y Rivero Weber, P.: El desafío de la bioética. México: Fondo de Cultura Económica, 2009, pp. 15 y ss.

32. Evers, K.: Neuroética. Cuando la materia se despierta, trad. de V. Goldstein. Madrid: Katz, 2010, p. 187.

33. Vid. Jonas, H.: El principio de responsabilidad, op. cit., pp. 230 y ss.

34. Sobre el tema vid. Romañach, J.: Bioética al otro lado del espejo. A Coruña: Diversitas, 2009 